Mathematical Modelling and Analysis

Volume 19 Number 3, June 2014, 359-370

http://dx.doi.org/10.3846/13926292.2014.925005

(c) Vilnius Gediminas Technical University, 2014
Publisher: Taylor\&Francis and VGTU

http://www.tandfonline.com/TMMA

Print ISSN: 1392-6292

Online ISSN: 1648-3510

\title{
On Onset of Chaotic Convection in Couple-Stress Fluids
}

\section{Jadallah M. Jawdat $^{a}$, Ishak Hashim ${ }^{a}$, Beer S. Bhadauria ${ }^{b}$ and Shaher Momani ${ }^{c, d}$}

a School of Mathematical Sciences, Universiti Kebangsaan Malaysia 43600 Bangi Selangor, Malaysia

${ }^{b}$ Department of Applied Mathamatics 6 Statistics, School for Physical

Sciences, Babasaheb Bhimrao Ambedkar University

Lucknow-226025, India

${ }^{c}$ Department of Mathematics, Faculty of Science, The University of Jordan

Amman 11942, Jordan

${ }^{d}$ Nonlinear Analysis and Applied Mathematics (NAAM) Research Group, Faculty of Science, King Abdulaziz University

Jeddah 21589, Saudi Arabia

E-mail(corresp.): jadjawdat@gmail.com

E-mail: ishak_h@ukm.my

E-mail: mathsbsb@yahoo.com

E-mail: s.momani@ju.edu.jo

Received March 29, 2013; revised May 6, 2014; published online June 1, 2014

\begin{abstract}
The effect of couple-stress fluid field on chaotic convection in a fluid layer heated from below was studied in this paper based on the theory of dynamical systems. A low-dimensional, Lorenz-like model was obtained using Galerkin truncated approximations. The fourth-order Runge-Kutta method was employed to solve the nonlinear system. The results show that inhibition of chaotic convection depends strongly on the couple-stress parameter.
\end{abstract}

Keywords: chaotic convection, couple-stress fluid, Lorenz equations.

AMS Subject Classification: 37D45.

\section{Introduction}

Chaotic convection has attracted interest due to its wide applications in many natural systems, such as the time evolution of the magnetic field of celestial bodies, molecular vibrations, the dynamics of satellite in the solar system, the weather, ecology and neurons.

The transition from steady convection to chaos for low Prandtl number was studied by Vadasz and Olek [19] is sudden and occurs by a subcritical 
Hopf bifurcation producing a solitary limit cycle which may be associated with a homoclinic explosion. This finding can be recovered from a truncated Galerkin expansion [18] that yields a system identical to the familiar Lorenz equations [6,12]. The work of Vadasz [15] suggests an explanation for the appearance of this solitary limit cycle via local analytical results. For the corresponding convection problem in a pure fluid, a similar approach was used by Vadasz $[16,17]$ to demonstrate similar results. Vadasz and Olek [20] showed that the route to chaos occurs by a period doubling sequence of bifurcations when the Prandtl number is moderate. Sheu [11] studied thermal convection in a fluid-saturated porous medium using a thermal non-equilibrium model to take account of the interphase heat transfer between the fluid and the solid. He found that the route to chaos was altered by interphase heat transfer and the non-equilibrium effect tends to stabilize steady convection. He also predicted an abrupt transition to chaos when interphase heat transfer is moderate and the porosity-modified conductivity ratio is small or moderate, while a perioddoubling route to chaos was predicted with weak interphase heat transfer and a small-porosity-modified conductivity ratio. Jawdat and Hashim [4] studied the chaotic convection in a porous medium for low Prandtl number with influence of internal heat generation. They showed that the onset of chaotic convection can be enhanced by a uniform internal heat generation. Whereas, the inhibition of chaotic convection for pure fluid using nanofluids was investigated by Jawdat et al. [5]. Idris and Hashim [3] observed that the magnetic field could delay the convective motion in a saturated porous medium fluid layer for low Prandtl number case. Meanwhile, Mahmud and Hashim [7] showed that a constant, vertical magnetic field could suppress or enhance the chaotic convection in a fluid layer heated from below.

Couple-stress fluids introduced by [13], is a special case of a non-Newtonian fluid and have distinct features, such as the presence of couple stresses, body couples and non-symmetric stress tensor. The main feature of couple stresses is to introduce a size dependent effect. The study of couple-stress fluids has applications in a number of processes that occur in industry such as the extrusion of polymer fluids, solidification of liquid crystals, cooling of metallic plate in a bath. The stability of a layer of fluid with couple stresses was studied by Ahmadi [1]. He observed that the critical Rayleigh number is a sharp limit separating the stability and instability region. The electrically conducting couple-stress fluid heated from below in porous medium in the presence of uniform magnetic field was studied by Sharma and Thakur [10]. They concluded that, for stationary convection, the couple stress and magnetic field postpone the onset of convection whereas the medium permeability hastens the onset of convection. Also, the magnetic field introduces oscillatory modes in the system which were non-existent in its absence. Devakar and Iyengar [2] studied Stokes first and second problems for an incompressible couple-stress fluid under isothermal conditions and observed that an increase in the couple-stress parameter has a decreasing influence on the velocity. Malashetty et al. [8] examined analytically the stability of a horizontal couple stress fluid saturated porous layer heated from below when the solid and fluid phases are not in local thermal equilibrium. They showed that the results of the thermal non- 
equilibrium Darcy model for the Newtonian fluid case can be recovered in the limit as couple stress parameter approaches zero. They also found an excellent agreement between the exact solutions and asymptotic solutions when the interphase heat transfer coefficient is very small. The nonlinear stability as well as linear instability analysis of couple-stress fluid heated from below was discussed by Sunil et al. [14]. They established that the linear instability and nonlinear stability Rayleigh numbers are the same. Further, they found that the couple-stress fluid is thermally more stable than the ordinary fluid. Saravanan and Premalatha [9] investigated the effect of couple stress on the onset of thermovibrational convection in a horizontal porous layer heated from one side. They found that the vibrating frequency can either augment or suppress the onset of convection in the case of bottom heating whereas always augments it in the case of top heating. In addition, they found that the presence of couple stress in the fluid restricts the ensuing synchronous convective pattern to lower frequencies in the case of bottom heating whereas extends it to higher frequencies in the case of top heating.

The aim of the present work is to extend the work of the work of Vadasz [17] to study the influence of couple-stress fluid on chaotic convection in a fluid layer heated from below. The truncated Galerkin approximation was applied to the governing equations to deduce an autonomous system with three ordinary differential equations. This system was used to investigate the dynamic behaviour of thermal convection in the fluid layer and to elucidate the effects of couplestress fluid on the transition to chaos.

\section{Problem Formulation}

Consider a very long and narrow fluid layer subject to gravity and heated from below, with the influence of couple-stress fluid. A Cartesian co-ordinate system is used such that the vertical axis $z$ is collinear with gravity, i.e. $\hat{e_{g}}=-\hat{e_{z}}$.

A linear relationship between density and temperature is assumed and can be presented as $\rho=\rho_{0}\left[1-\beta_{*}\left(T_{*}-T_{c}\right)\right]$, where $\beta_{*}$ represents the thermal expansion coefficient. Also, the Boussinesq approximation is applied indicating that density variations are effected only for the gravity term in the momentum equation.

Subject to these conditions, the dimensionless governing equations can be written as

$$
\begin{aligned}
& \nabla \cdot V=0, \\
& \frac{1}{\operatorname{Pr}}\left[\frac{\partial}{\partial t}+V \cdot \nabla\right] V=\frac{-1}{\operatorname{Pr}} \nabla p+\nabla^{2} V-C \nabla^{4} V+R a T \hat{e_{z}}, \\
& \frac{\partial T}{\partial t}+V \cdot \nabla T=\nabla^{2} T,
\end{aligned}
$$

where $V$ is the velocity, $T$ is temperature, $p$ is pressure, $R a$ is the Rayleigh number defined in the form $R a=\beta_{*} \Delta T_{c} g_{*} H_{*}^{3} / \alpha_{*} \nu_{*}, \operatorname{Pr}$ is the Prandtl number defined by $\operatorname{Pr}=\nu_{*} / \alpha_{*}, C$ is the couple-stress parameter defined by $C=$ $\mu_{1} / \mu H_{*}^{2}, \mu$ is dynamic viscosity, $\mu_{1}$ is couple-stress viscosity. The values $\alpha_{*} / H_{*}$, 
$\rho_{0} \alpha_{*}^{2} / H_{*}^{2}$, and $\Delta T_{c}=\left(T_{H}-T_{C}\right)$ were used to scale the velocity components $\left(u_{*}, v_{*}, w_{*}\right)$, pressure $\left(p_{*}\right)$, and temperature variations $\left(T_{*}-T_{C}\right)$, respectively, where $\alpha_{*}$ is the thermal diffusivity and $\rho_{0}$ is a reference value of density. The height of the layer $H_{*}$ was used for scaling the variables $x_{*}, y_{*}, z_{*}$ and $H_{*}^{2} / \alpha_{*}$ for scaling the time $t_{*}$. Accordingly, $x=x_{*} / H_{*}, y=y_{*} / H_{*}, z=z_{*} / H_{*}$ and $t=t_{*} \alpha_{*} / H_{*}^{2}$.

The fluid layer with stress-free (i.e. no tangential shear stress) horizontal boundaries is considered. Hence, the solution must follow the impermeability conditions $V \cdot \hat{e}_{n}=0$ and the stress free condition $\partial u / \partial z=\partial v / \partial z=\partial^{2} w / \partial z^{2}=$ 0 on these boundaries, where $\hat{e}_{n}$ is a unit vector normal to the boundary. The temperature boundary conditions are: $T=1$ at $z=0, T=0$ at $z=1$.

The governing equations can be represented in terms of a stream function defined by $u=-\partial \psi / \partial z$ and $w=\partial \psi / \partial x$, as for convective rolls having axes parallel to the shorter dimension (i.e. $y$ ) when $v=0$. Applying the curl $(\nabla \times)$ operator on Eq. (2.2) yields the following system of partial differential equations from Eqs. (2.1)-(2.3):

$$
\begin{aligned}
& {\left[\frac{1}{\operatorname{Pr}}\left(\frac{\partial}{\partial t}-\frac{\partial \psi}{\partial z} \frac{\partial}{\partial x}+\frac{\partial \psi}{\partial x} \frac{\partial}{\partial z}\right)-\nabla^{2}+C \nabla^{4}\right]\left(\nabla^{2} \psi\right)=R a\left(\frac{\partial T}{\partial x}\right),} \\
& \frac{\partial T}{\partial t}-\frac{\partial \psi}{\partial z} \frac{\partial T}{\partial x}+\frac{\partial \psi}{\partial x} \frac{\partial T}{\partial z}=\frac{\partial^{2} T}{\partial x^{2}}+\frac{\partial^{2} T}{\partial z^{2}} .
\end{aligned}
$$

The boundary conditions for the stream function are $\psi=\partial \psi / \partial z=0$ on all horizontal boundaries and the couple stresses vanish at the boundary.

The set of partial differential equations, (2.4) and (2.5), form a nonlinear coupled system and together with the corresponding boundary conditions will accept a basic motionless conduction solution.

\section{Reduced Set of Equations}

In order to obtain the solution to the nonlinear coupled system of partial differential equations in (2.4) and (2.5), the stream function and temperature are represented in the form

$$
\psi=A_{11} \sin (\kappa x) \sin (\pi z), \quad T=1-z+B_{11} \cos (\kappa x) \sin (\pi z)+B_{02} \sin (2 \pi z) .
$$

This representation is equivalent to a Galerkin expansion of the solution in both the $x$ - and $z$-directions. Unlike in the works of Vadasz [17], the time and amplitudes are rescaled with respect to their convective fixed points of the form

$$
X=\frac{\tilde{A}_{11}}{\sqrt{\lambda \frac{(R-S)}{S}}}, \quad Y=\frac{\tilde{B}_{11}}{S \sqrt{\lambda \frac{(R-S)}{S}}}, \quad Z=\frac{-\tilde{B}_{02}}{(R-S)}
$$

to have the following system of ordinary differential equations

$$
\begin{aligned}
& \dot{X}=S \operatorname{Pr}(Y-X), \\
& \dot{Y}=\left(\frac{R}{S}\right) X-Y-\left(\frac{R-S}{S}\right) X Z, \\
& \dot{Z}=\lambda(X Y-Z),
\end{aligned}
$$


where

$$
\begin{aligned}
& R=\frac{R a}{R a_{c}}, \quad R a_{c}=\frac{\left(\kappa^{2}+\pi^{2}\right)^{3}}{\kappa^{2}}, \quad \lambda=\frac{8}{\left[\left(\kappa / \kappa_{c r}\right)^{2}+2\right]}, \\
& \tau=\left(\kappa^{2}+\pi^{2}\right) t, \quad \kappa_{c r}=\frac{\pi}{\sqrt{2}}, \quad S=1+\frac{5 \pi^{2}}{6} C, \\
& \tilde{A}_{11}=\frac{\left(\kappa / \kappa_{c r}\right)}{\left[\left(\kappa / \kappa_{c r}\right)^{2}+2\right]} A_{11}, \quad \tilde{B}_{11}=\kappa_{c r} R B_{11}, \quad \tilde{B}_{02}=\pi R B_{02}
\end{aligned}
$$

and the primes () denote time derivatives $\mathrm{d}() / \mathrm{d} \tau$. When $C=0$, (i.e. $S=1$ ), system (3.1)-(3.3) reduces to the Vadasz system [17] (Eqs. (11)-(13)). System (3.1)-(3.3) is equivalent to the Lorenz equations [6,12], although with different coefficients. By using the wavenumber corresponding to the convection threshold, i.e. $\kappa_{c r}$, in the definitions of $\lambda$ and $R a_{c}$ (Eq. (3.4)) yields $\lambda=8 / 3$ and $R a_{c}=27 \pi^{4} / 4$.

\section{Stability Analysis}

Stability analysis of the stationary solutions was performed in order to determine the nature of the dynamics of the fixed points. The nonlinear dynamics of a Lorenz-like system (3.1)-(3.3) has been analyzed and solved for $\operatorname{Pr}=10$ and $\lambda=8 / 3$. This rescaled system has three fixed points.

The first fixed point is $X_{1}=Y_{1}=Z_{1}=0$, corresponding to the motionless solution, while the second and the third fixed points corresponding to the convection solution are $X_{2,3}=Y_{2,3}= \pm 1, Z_{2,3}=1$.

The stability of the first fixed point, $X_{1}=Y_{1}=Z_{1}=0$, is controlled by the zeros of the following characteristic polynomial equation for the eigenvalues $\alpha_{i}$ $(i=1,2,3)$ :

$$
(-\lambda-\alpha)[(S \operatorname{Pr}+\alpha)(1+\alpha)-\operatorname{Pr} R]=0 .
$$

The first eigenvalue, $\alpha_{1}=-\lambda$, is negative since $\lambda=8 / 3$. The other two eigenvalues are always real and given by

$$
\alpha_{2,3}=\frac{1}{2}\left[-(1+S P r) \pm \sqrt{(1+S P r)^{2}+4 \operatorname{Pr}(R-S)}\right]
$$

$\alpha_{3}$ is also negative and $\alpha_{2}$ provides the stability condition for the motionless solution in the form $\alpha_{2}<0 \Leftrightarrow R<S$. Therefore, the critical value of $R$, where the motionless solution loses stability and the convection solution (expressed by the other two fixed points) takes over, is obtained as

$$
R_{c 1}=R_{c r}=S,
$$

which corresponds to $R a_{c r}=\left(27 \pi^{4} / 4\right) S$. This means that there is a direct proportion between the couple-stress parameter $C$ and the Rayleigh number $R a$ as in Fig. 1.

The following cubic equation for the eigenvalues, $\alpha_{i}(i=1,2,3)$, controls the stability of the second and the third fixed points of the rescaled system

$$
\alpha^{3}+(1+\lambda+S P r) \alpha^{2}+\left(S P r+R S^{-1}\right) \lambda \alpha+2 \operatorname{Pr} \lambda(R-S)=0 .
$$




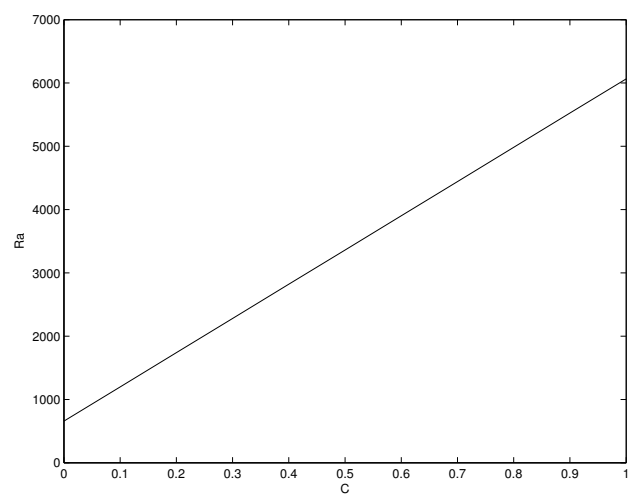

Figure 1. A graph of $C$ and $R a$ showing the direct proportionality between them.

Eq. (4.2) yields three eigenvalues, and the smallest of which $\alpha_{1}$ is always real and negative over the whole range of parameter values. The other two are real and negative at slightly supercritical values of $R$, such that the convection fixed points are stable, that is, simple nodes. These two roots move on the real axis towards the origin as the value of $R$ increases. For $\operatorname{Pr}=10$ and $\lambda=8 / 3$, these roots become equal when $R \cong 2.445$ for $C=0.1$ and $R \cong 3.54$ for $C=0.2$ compared with $R \cong 1.35$ for $C=0$ (Vadasz case). It is exactly at this point that these two roots become a complex conjugate. In any case, they still have negative real parts, and so the convection fixed points are stable, that is, spiral nodes. Both the imaginary and real parts of these two complex conjugate eigenvalues increase and extend over the imaginary axis as the value of $R$ increases. The real part becomes nonnegative at a value of $R$ given by

$$
R_{c 2}=\frac{S^{2} \operatorname{Pr}(3+\lambda+S \operatorname{Pr})}{(S \operatorname{Pr}-\lambda-1)} .
$$

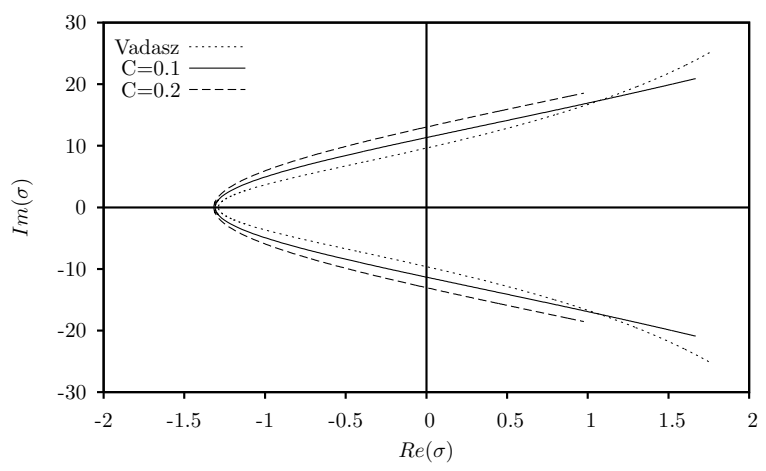

Figure 2. Evolution of the complex eigenvalues with increasing Rayleigh number, for $\operatorname{Pr}=10, \lambda=8 / 3$, Vadasz case, $C=0.1$ and $C=0.2$.

Relation (4.3) is an extension of $R_{0}$ in [17] to the couple stress case $C \neq 0$ (i.e. $S \neq 1$ ). At this point, the convection fixed points lose their stability 
and other (periodic or chaotic) solutions take over. The loss of stability of the convection fixed points for $\operatorname{Pr}=10$ and $\lambda=8 / 3$ using Eq. (4.3) are evaluated to be $R_{c 2}=54.50771700$ for $C=0.1$ and $R_{c 2}=98.61581171$ for $C=0.2$ compared with $R_{0}=24.737$ for Vadasz loss of stability of the convection fixed points when $C=0$. For $\operatorname{Pr}=10, \lambda=8 / 3, C=0.1$ and $C=0.2$, the evolutions of the complex eigenvalues are presented in Fig. 2.

\section{Results and Discussion}

In this section, some numerical simulations of the system (3.1)-(3.3) are presented for the time domain $0 \leq \tau \leq 210$. All calculations were done using MATLAB's built-in ODE45 based on the fourth-order Runge-Kutta method on double precision with stepsize 0.001, fixing the values $\operatorname{Pr}=10$ and $\lambda=8 / 3$, and taking the initial conditions $X(0)=Y(0)=0.8$ and $Z(0)=0.92195$.

Also, the bifurcation diagrams are presented in Fig. 3, in terms of maxima and minima in the post-transient values of $Z$ versus $R$ for $C=0$ (Vadasz case), $C=0.1$ and $C=0.2$ with $15 \leq R \leq 450$ and a resolution of $\Delta R=0.25$.

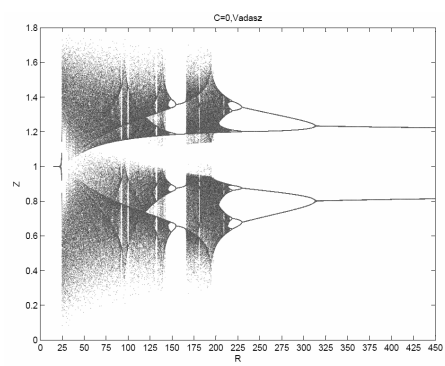

(a)

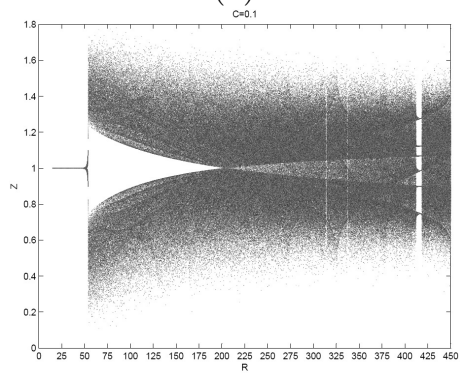

(c)

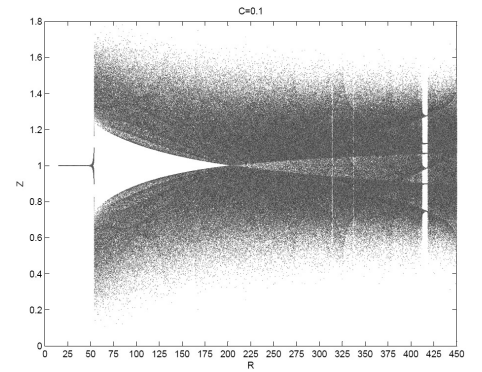

(b)

Figure 3. Bifurcation diagrams of $Z$ versus $R$ representing maxima and minima of the post-transient solution of $Z(t)$ for $\operatorname{Pr}=10, \lambda=8 / 3$, (a) $C=0$ Vadasz case, (b) $C=0.1$ and (c) $C=0.2$.

It is observed from Fig. 3 that the chaotic behaviour is delayed with increasing the chaotic region when using the couple-stress fluid.

A comparison between Vadasz case and the cases under study is displayed in Table 1 for $R$ is a solitary limit cycle, signifying the loss of stability of the 
steady convection fixed points and the critical value of $R$ at which the chaotic behaviour solution occurs.

Table 1. Comparison between Vadasz case $(C=0)$ and the couple-stress case for $C=0.1$ and $C=0.2$ with $\operatorname{Pr}=10, \lambda=8 / 3$ for $R$ a solitary limit cycle signifying the loss of stability of the steady convection fixed points and the critical value of $R$ at which the chaotic behaviour solution occurs.

\begin{tabular}{lll}
\hline$C$ & $\begin{array}{l}R \\
(\text { limit cycle) }\end{array}$ & $\begin{array}{l}R \\
\text { (critical value, chaotic behaviour) }\end{array}$ \\
\hline$C=0$ (Vadasz) & 24.4207 & 24.737 \\
$C=0.1$ & 53.81 & 54.50771700 \\
$C=0.2$ & 97.3038 & 98.61581171 \\
\hline
\end{tabular}

Further, a comparison between Vadasz case and the couple stress case is mentioned in Table 2 for the critical value of $R$ at which the chaotic behaviour solution occurs.

Table 2. Comparison between Vadasz case $(C=0)$ and the couple-stress case $(C \neq 0)$ with $\operatorname{Pr}=10, \lambda=8 / 3$ for the critical value of $R$ at which the chaotic behaviour solution occurs.

\begin{tabular}{lll}
\hline$C$ & $S$ & $\begin{array}{l}R \\
\text { (critical value, chaotic behaviour) }\end{array}$ \\
\hline$C=0$ (Vadasz) & 1 & 24.737 \\
$C=0.1$ & 1.822467034 & 54.50771700 \\
$C=0.2$ & 2.644934068 & 98.61581171 \\
$C=0.3$ & 3.467401101 & 156.4180196 \\
$C=0.4$ & 4.289868134 & 227.8105223 \\
$C=0.5$ & 5.112335169 & 312.7614720 \\
$C=0.6$ & 5.934802202 & 411.2578375 \\
$C=0.7$ & 6.757269235 & 523.2932968 \\
$C=0.8$ & 7.579736270 & 648.8644104 \\
$C=0.9$ & 8.402203303 & 787.9691482 \\
$C=1.0$ & 9.224670336 & 940.6062324 \\
\hline
\end{tabular}

Comparing to Vadasz case [17], and referring to Table 2, it is obvious that the critical value of $R$ in each case is greater than the critical value in Vadasz case (in huge manner). Thus, the onset of chaotic convection is strongly delayed. More details are presented below for $C=0.1$ and $C=0.2$.

\subsection{Case $C=0.1$}

In the case of $C=0.1$, it is found that at $R_{c 1}=1.822467034$, obtained from Eq. (4.1), the motionless solution loses stability and the convection solution takes over. Moreover, the values of the eigenvalues $\alpha_{2}$ and $\alpha_{3}$ from Eq. (4.2) become equal and complex conjugate when $R \cong 2.445$. At the value of $R=53.81$, a solitary limit cycle signifying the loss of stability of the steady convection fixed points are obtained. When $R=54.507717$, the convection fixed points 
(a)

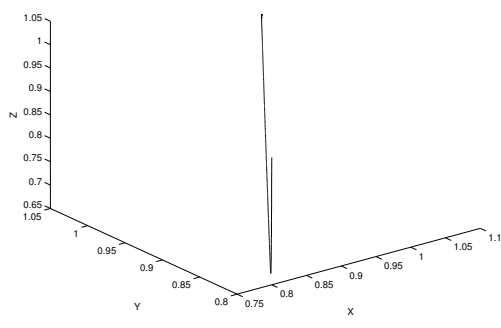

$\{\mathrm{C}=0.1, \mathrm{R}=53)$

(c)

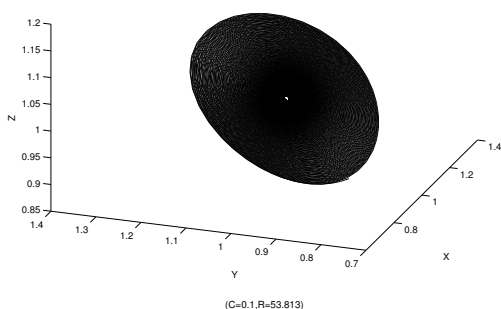

(e)

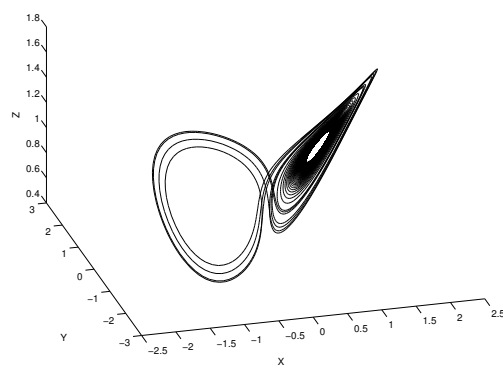

(b)

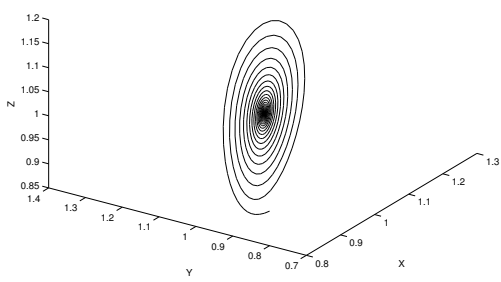

$(\mathrm{C}=0.1 \mathrm{R}=53.81)$

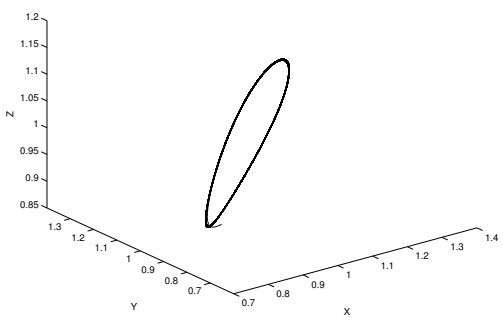

(C=0.1,R=54.507717)

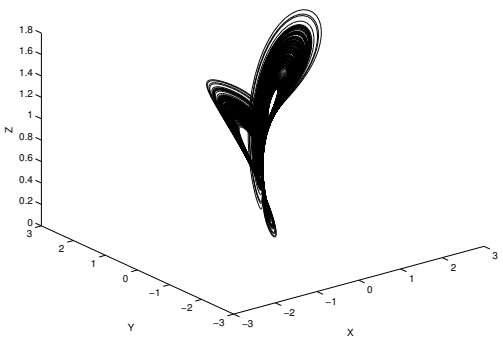

Figure 4. Computational results for the evolution of trajectories over time in the state space for increasing values of scaled Rayleigh number $(R)$. The graphs represent the projection of the solution data points onto $X-Y-Z$ plane for $\operatorname{Pr}=10, \lambda=8 / 3$ and $C=0.1$ for (a) $R=1.9$, (b) $R=40$, (c) $R=53$, (d) $R=53.81$, (e) $R=53.813$, (f) $R=54.50771700$.

lose their stability and a chaotic solution takes over. The evolution of trajectories over time in the state space for two values of Rayleigh number $R$, where the solution is limit cycle and the critical value of $R$, is presented in Fig. 4.

\subsection{Case $C=0.2$}

In this case of study $(C=0.2)$, it is found that at $R_{c 1}=2.644934068$, obtained from Eq. (4.1), the motionless solution loses stability and the convection solution takes over. Also, the values of the eigenvalues $\alpha_{2}$ and $\alpha_{3}$ from Eq. (4.2) become equal and complex conjugate when $R \cong 3.54$. At the value of $R=97.3038$, a solitary limit cycle signifying the loss of stability of the steady convection fixed points are obtained. When $R=98.61581171$, the convection fixed points lose their stability and a chaotic solution takes over. The evolution 
(a)

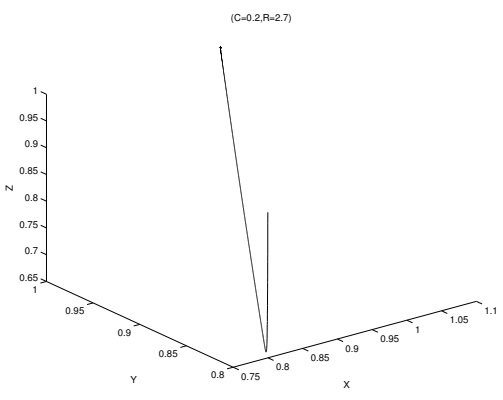

(b)

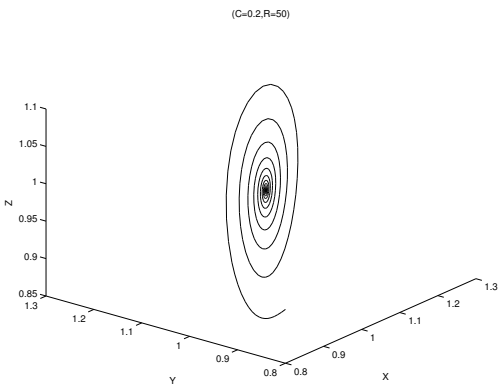

( $\mathrm{C}=0.2, \mathrm{R}=97.3038)$

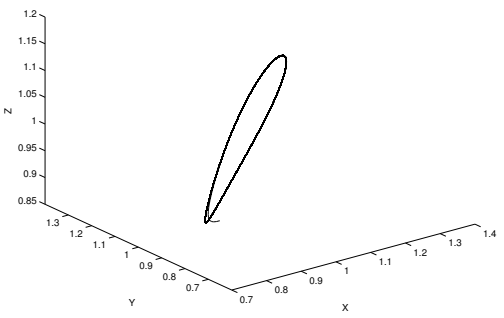

(d)

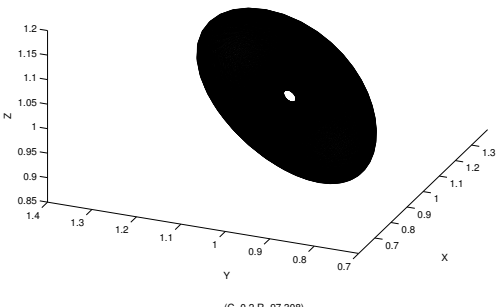

(c) $(\mathrm{C}=0.2, \mathrm{R}=97.308)$

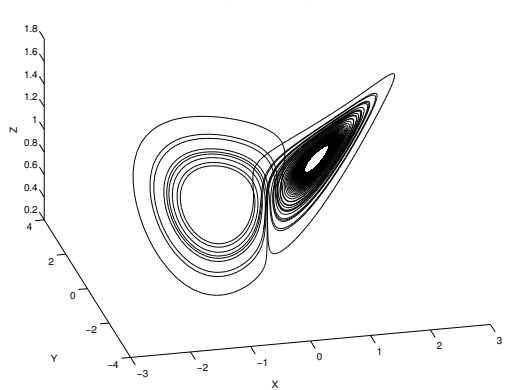

(e)

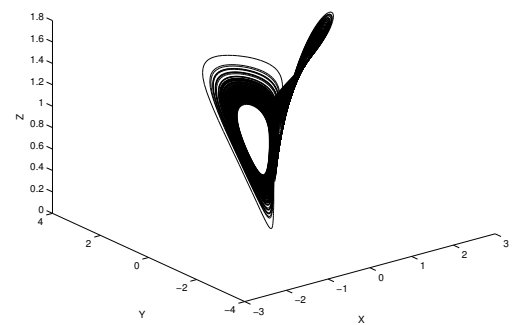

Figure 5. Computational results for the evolution of trajectories over time in the state space for increasing values of scaled Rayleigh number $(R)$. The graphs represent the projection of the solution data points onto $X-Y-Z$ plane for $\operatorname{Pr}=10, \lambda=8 / 3$ and $C=0.2$ for for (a) $R=2.7$, (b) $R=50$, (c) $R=97$, (d) $R=97.3038$, (e) $R=97.308$, (f) $R=98.61581171$.

of trajectories over time in the state space is presented in Fig. 5 for two values of Rayleigh number $R$, where the solution is limit cycle and the critical value of $R$.

\section{Conclusions}

In this paper, chaotic behaviour in a fluid layer subject to gravity and heated from below under the effect of couple-stress fluid are studied. It is noticed that there is a direct proportion between the couple-stress parameter $C$ and the Rayleigh number $R a$. In comparison with Vadasz case, it is concluded that the onset of chaotic convection can be strongly delayed under the influence of 
couple-stress fluid. Thus, the stability region can be strongly increased under the influence of couple-stress fluid.

\section{References}

[1] G. Ahmadi. Stability of a cosserat fluid layer heated from below. Acta Mech., 31:243-252, 1979. http://dx.doi.org/10.1007/BF01176852.

[2] M. Devakar and T.K.V. Iyengar. Stokes' problems for an incompressible couple stress fluid. Nonlinear Anal. Model. Control, 1(2):181-190, 2008.

[3] R. Idris and I. Hashim. Effects of a magnetic field on chaos for low Prandtl number convection in porous media. Nonlinear Dynam., 62:905-917, 2010. http://dx.doi.org/10.1007/s11071-010-9773-8.

[4] J.M. Jawdat and I. Hashim. Low Prandtl number chaotic convection in porous media with uniform internal heat generation. International Communication in Heat and Mass Transfer, 37:629-636, 2010. http://dx.doi.org/10.1016/j.icheatmasstransfer.2010.03.011.

[5] J.M. Jawdat, I. Hashim and S. Momani. Dynamical system analysis of thermal convection in a horizontal layer of nanofluids heated from below. Math. Probl. Eng., 2012, 2012. http://dx.doi.org/10.1155/2012/128943.

[6] E.N. Lorenz. Deterministic nonperiodic flow. J. Atmospheric Sci., 20:130-141, 1963. http://dx.doi.org/10.1175/1520-0469(1963)020¡0130:DNF ¿2.0.CO;2.

[7] M.N. Mahmud and I. Hashim. Effects of magnetic field on chaotic convection in fluid layer heated from below. International Communication in Heat and Mass Transfer, 38:481-486, 2011.

http://dx.doi.org/10.1016/j.icheatmasstransfer.2010.12.023.

[8] M.S. Malashetty, I.S. Shivakumarab and S. Kulkarni. The onset of convection in a couple stress fluid saturated porous layer using a thermal non-equilibrium model. Phys. Lett. A, 373:781-790, 2009.

http://dx.doi.org/10.1016/j.physleta.2008.12.057.

[9] S. Saravanan and D. Premalatha. Effect of couple stress on the onset of thermovibrational convection in a porous medium. International Journal of Thermal Sciences, 57:71-77, 2012. http://dx.doi.org/10.1016/j.ijthermalsci.2012.02.013.

[10] R.C. Sharma and K.D. Thakur. On couple-stress fluid heated from below in porous medium in hydromagnetics. Czechoslovak J. Phys., 50:753-758, 2000. http://dx.doi.org/10.1023/A:1022886903213.

[11] L.J. Sheu. An autonomous system for chaotic convection in a porous medium using a thermal non-equilibrium model. Chaos Solitons Fractals, 30:672-689, 2006. http://dx.doi.org/10.1016/j.chaos.2005.11.080.

[12] C. Sparrow. The Lorenz Equations: Bifurcations, Chaos and Strange Attractors. Springer-Verlag, New York, 1982.

[13] V.K. Stokes. Couple stresses in fluids. Phys. Fluids, 9:1709-1715, 1966. http://dx.doi.org/10.1063/1.1761925.

[14] Sunil, R. Devi and A. Mahajan. Global stability for thermal convection in a couple-stress fluid. International Communication in Heat and Mass Transfer, 38:938-942, 2011. http://dx.doi.org/10.1016/j.icheatmasstransfer.2011.03.030. 
[15] P. Vadasz. Local and global transitions to chaos and hysteresis in a porous layer heated from below. Transp. Porous Media, 37:213-245, 1999. http://dx.doi.org/10.1023/A:1006658726309.

[16] P. Vadasz. On the homoclinic orbit for convection in a fluid layer heated from below. International Journal of Heat and Mass Transfer, 42:3557-3561, 1999. http://dx.doi.org/10.1016/S0017-9310(99)00042-3.

[17] P. Vadasz. Subcritical transitions to chaos and hysteresis in a fluid layer heated from below. International Journal of Heat and Mass Transfer, 43:705-724, 2000. http://dx.doi.org/10.1016/S0017-9310(99)00173-8.

[18] P. Vadasz and S. Olek. Transitions and chaos for free convection in a rotating porous layer. International Journal of Heat and Mass Transfer, 41:1417-1435, 1999. http://dx.doi.org/10.1016/S0017-9310(97)00265-2.

[19] P. Vadasz and S. Olek. Weak turbulence and chaos for low Prandtl number gravity driven convection in porous media. Transp. Porous Media, 37:69-91, 1999. http://dx.doi.org/10.1023/A:1006522018375.

[20] P. Vadasz and S. Olek. Route to chaos for moderate Prandtl number convection in a porous layer heated from below. Transp. Porous Media, 41:211-239, 2000. http://dx.doi.org/10.1023/A:1006685205521. 